\title{
A Novel Switched Capacitor Technique for NBTI Tolerant Low Power 6T-SRAM Cell Design
}

\author{
Ashvinikumar Dongre ${ }^{1}$, Manisha Pattanaik ${ }^{1}$, Balwinder Raj ${ }^{2}$, Arti Naik ${ }^{3}$ \\ IVLSI Design Lab, ABV-IIITM Gwalior, India. \\ 2Dept. of ECE, NIT Jalandhar, Punjab, India. \\ 3Dept of computer engineering SKNCOE, Pune, India.
}

\begin{abstract}
The work in this paper is focused on designing a NBTI tolerant 6T SRAM cell while maintaining low power operation. We explore the usefulness of switched capacitor circuit to provide NBTI tolerance while reducing overall power consumption. A thorough analysis of the 6T SRAM cell has been done to show the reduction in power consumption of the cell without degrading the read and write stability. The results obtained in proposed technique are compared and contrasted with reported data for the validation of our approach. The proposed technique reduces read power by $32 \%$, write power by $15 \%$ and leakage power by $14 \%$. The applied technique effectively reduces overall current by considerable amount and also significant reduction in Vth degradation due to NBTI is observed hence it is suitable for NBTI tolerant low power 6T SRAM cell design.
\end{abstract}

Keywords: NBTI, Low Power, 6T SRAM cell, Switched capacitor, SNM.

\section{Introduction}

As the circuit complexity of day to day applications is increasing at a rapid speed, memory is emerging as the most important factor in deciding the performance of any system. Larger the memory available better will be the performance. The modern video processors have to do a lot of complex computations which can be done at faster rate if more memory is available. The processing speed of a processor is often limited by the amount of cache being used, thus more and more memory need to be added on a single chip to improve system performance, but at the same time it has been found that most of the power is being dissipated by the memory alone. Most of the times the power consumed by the memory becomes more than the logic circuit present on the chip. Thus memories have become the epicenter of power consumption in modern systems [1].

On the other side the trend of reducing the transistor feature size continues, process variation in devices has made it very difficult to reduce the cell area at 50\% rate. Among the entire different device variations Negative Bias Temperature Instability (NBTI) is found to affect the device reliability more severely and thus in recent years have become major reliability issue for the semiconductor industry. NBTI impact is getting worse in each technology generation with greater performance and reliability loss. Register's and caches which are SRAM cells, are also much severely affected due to the NBTI aging. If an SRAM cell stores same value for a large period of time it goes through high amount of stress, thus reducing its reliability characteristics which will in return result in a loss of the stored data. Therefore, register files and caches have become more prone to failures due to NBTI. Remaining part of the paper is arranged as follows, section-2 gives a brief about basic 6T SRAM cell operation, section-3 describes the proposed technique in detail, the obtained results are discussed in section4 , and section-5 concludes the paper.

\section{Sram Review}

SRAM has been the core area of study since decades and many techniques have been continuously developed to meet the power requirements. In reported paper [2] authors have discussed sleep stack technique wherein multiple cell voltages have been used to reduce the leakage power. Careful selection of cell voltages reduces the leakage current up to a certain extent, also in [15] dynamic voltage scaling been discussed which says that during a fixed period of time the activity in a cache is only centered on a small subset of the lines. This behavior has been exploited to cut the leakage power of large caches by putting the cold cache lines into a state preserving low-power drowsy mode but Moving lines into and out of drowsy state incurs a slight performance loss [13].

Today, SRAM cell needs to be designed not only with minimum area or high density but with as less power consumption as possible. Thus main focus in this research work is to reduce the overall power consumption of the SRAM cell. The proposed technique uses the switched capacitors to accumulate the charge flowing in the circuit during read and write cycles and when sufficient amount of charge is accumulated the SRAM is feed through the Switched capacitors instead of VDD, thus reusing the accumulated charge. 
2.1 SRAM 6T cell Basic operations

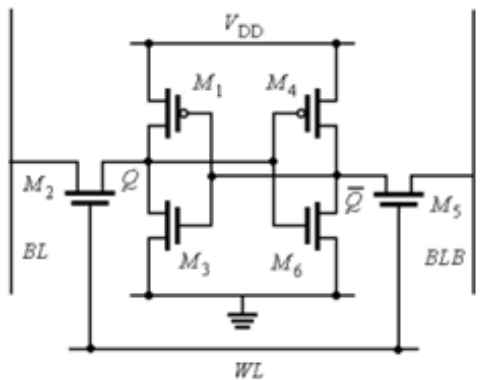

Figure 1: 6T SRAM Cell

Standby operation: When WL=0, M2 and M5 disconnect the cell from Bitlines. The two cross-coupled inverters formed by M1-M3 and M4-M6 will continue to reinforce each other as long as they are disconnected from bitlines.

Read operation: Read-1 cycle starts with pre-charging BL and BL to 1. Within the memory cell M1 and M6 are $\mathrm{ON}$. Asserting the word line, turns ON M2 and M5 and the values of Q and QB are transferred to Bit-Lines (BL and BLB). No current flows through M2, thus M1 and M2 pull BL up to VDD that is BL=1.BLB discharges through M5 and M6.

Write operation: The value to be written is applied to the Bit lines. Thus to write data 0 , we assert $\mathrm{BL}=0, \mathrm{BLB}=$ 1 and to write data 1 , the $\mathrm{BL}=1, \mathrm{BLB}=0$, asserted when $\mathrm{WL}=1$.

2.2 Switched Capacitor: A switched capacitor is electronic circuit which works by moving charges into and out of capacitors when switches are opened and closed. Usually, non-overlapping signals are used to control the switches, so that not all switches are closed simultaneously [9]. The switched capacitor diagram is as shown in Figure 2.

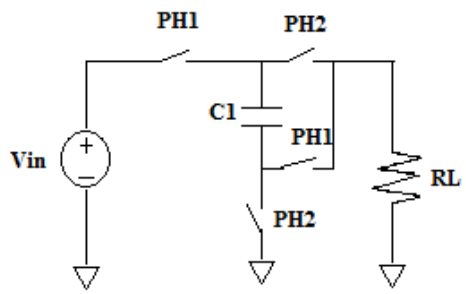

Figure 2: Switched Capacitor

During phase-1(PH-1), capacitor $\mathrm{C} 1$ is connected between the input node Vin and the output resistor RL. The charge flows from Vin goes though $\mathrm{C} 1$ and finally reaches the load resistor RL thus the capacitor gets charged up during phase-1. In phase-2(PH-2) $\mathrm{C} 1$ is connected between RL and GND, and hence now the charge stored previously on capacitor $\mathrm{C} 1$ drives the load resistor RL.

\subsection{Proposed SRAM with Switched Capacitor}

\section{Proposed Technique}

In the proposed technique the switched capacitor is connected in between the SRAM cell and the ground terminal. So that the charge flowing during read and write cycles would otherwise flow directly to the ground will now flow through the switched capacitor. As a result the capacitor will get charged and when a sufficient charge is accumulated this switched capacitor will replace the supply voltage VDD and will drive the SRAM cell. SRAM with switched capacitor is shown in Figure 3. Thus as shown in Figure 3, during phase-1 switches PH-1 will be turned on and the cell is operating at VDD=1V, and also the switched capacitor will get charged during this phase. 


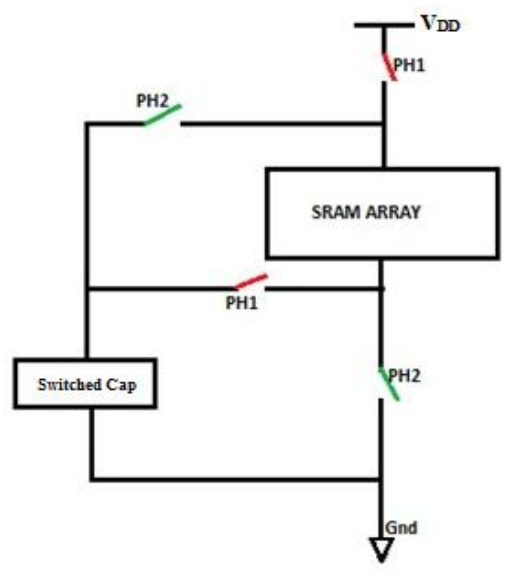

Figure 3: SRAM with switched capacitor

Phase-2 starts as soon as sufficient charge is accumulated and a sufficient voltage is obtained across the switched capacitor. Thus turning ON switches PH-2, now the switched capacitor circuit will drive the SRAM cell during idle state. Now in the idle state the SRAM is operating on voltage that is generated from the charge that would have been wasted in case switched capacitors were not used.

\subsection{Switched capacitor design:}

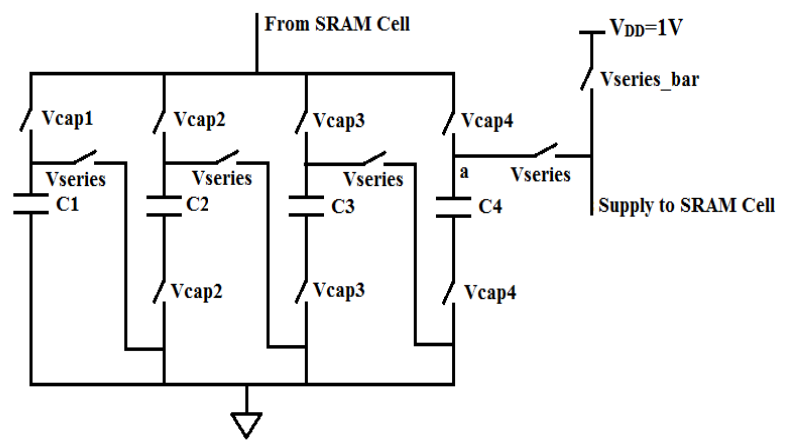

Figure 4: Switched Capacitor Arrangement

Initially Vseries is OFF, at the same time Vseries_bar is in ON condition, thus SRAM cell gets power from VDD. Now at this point Vcap1 is turned on therefore the read or write current depending on SRAM operation flows through SRAM Cell passes through capacitor C1 resulting in rise in capacitor voltage up to $0.2 \mathrm{~V}$. As soon as the capacitor voltage reaches $0.2 \mathrm{~V}$ Vcap1 is turned OFF and Vcap2 is turns ON thus now charging capacitor $\mathrm{C} 2$. This will continue till all four capacitors get charged. As soon as voltage across the fourth capacitor $\mathrm{C} 4$ reaches $0.2 \mathrm{~V}$, Vseries is turned $\mathrm{ON}$ resulting in connection of all the capacitors in series thus adding the potential across each one of them. If the switches are assumed to be ideal the total potential at point ' $\mathrm{a}$ ' will be $0.8 \mathrm{~V}$. But due to the drop across the transistors the voltage obtained at this point is $0.6 \mathrm{~V}$. Now the switched capacitor is ready to drive the SRAM Cell, so Vseries is turned ON, the capacitors get connected in series and now whenever the Cell goes in idle state the switched capacitor will drive the cell instead of VDD and the potential at terminals $\mathrm{Q}$ if it stores ' 1 ' will be $0.6 \mathrm{~V}$ instead of $1 \mathrm{~V}$. Thus reducing the standby power by $14.6 \%$.

\section{Results And Discussion}

The simulations were done at $65 \mathrm{~nm}$ technology with $\mathrm{VDD}=1 \mathrm{~V}$ and $\mathrm{Vth}=0.36 \mathrm{~V}$. As can be seen in Figure 5(a)-(d) the capacitors c1-c4 gets charged to a voltage of 0.2V. Figure 6(b) shows the series signal which connects the capacitors in series and also disconnects the cell from the VDD and drives the cell during idle state. Figure 6(c) clearly shows when Vseries is $\mathrm{ON}$ the cell goes into idle state and V(Q) falls to $0.6 \mathrm{~V}$ which is supplied from the switched capacitor, thus reducing leakage current. 


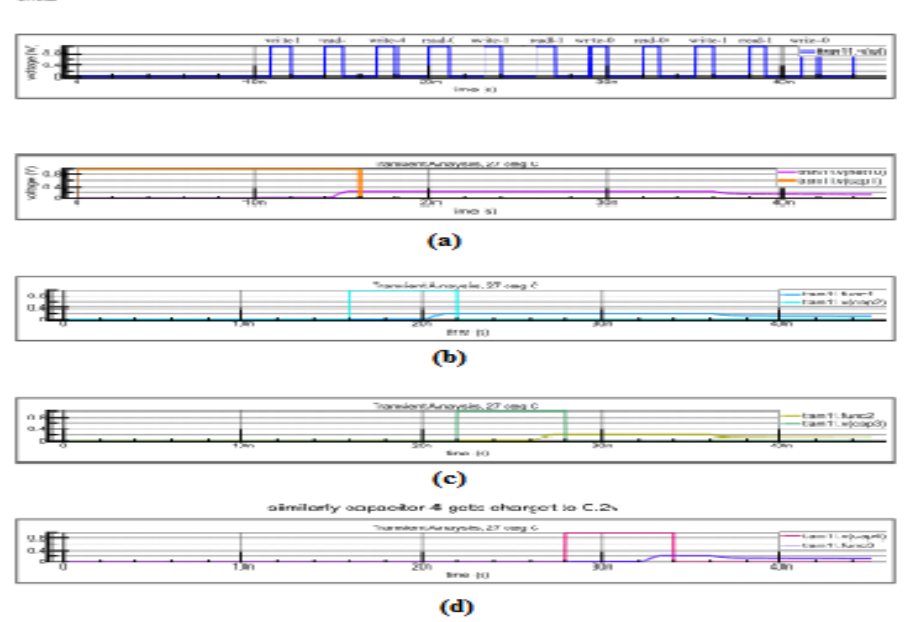

Figure 5 : Charging of capacitors during read and write operations

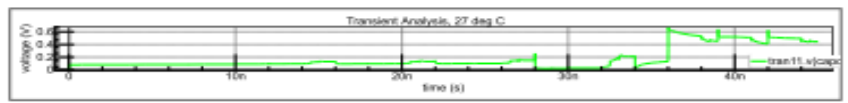

(a)

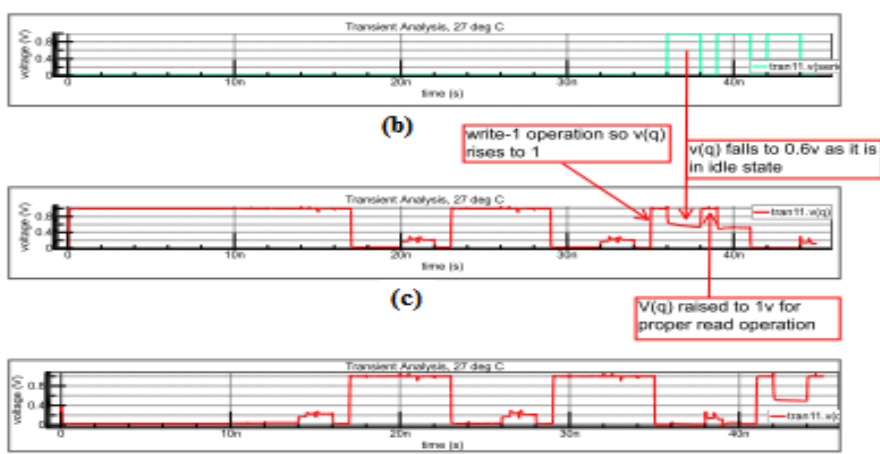

(d)

Figure $6:$ Switched capacitor drives the cell in idle state

\subsection{Read, Write and Leakage current:}

Figure 7 (a) and (b) shows the comparison of write- 1 and write- 0 currents in conventional SRAM cell and Cell with switched capacitor circuit respectively,as the supply voltage reduces the drain to source voltage reduces which results in reduction of the write current as can be observed in these Figure 7.

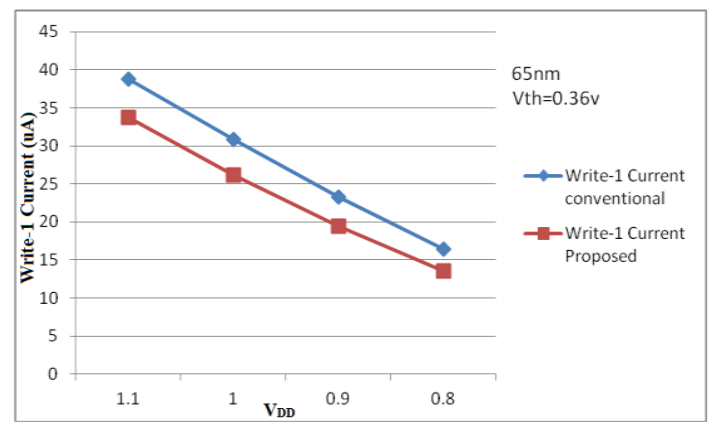

(a)

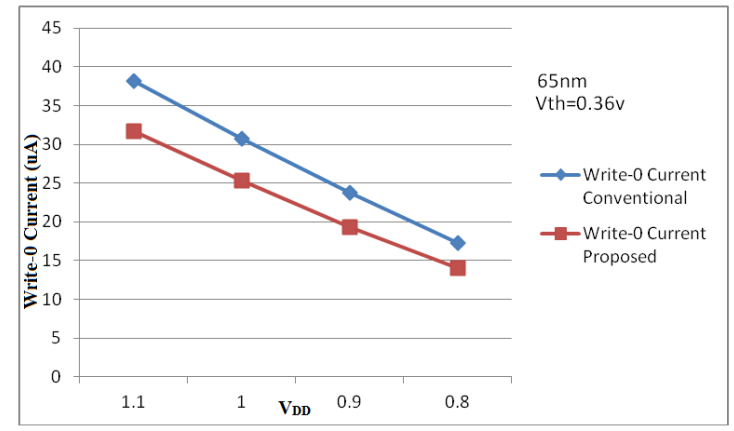

(b)

Figure 7 : Comparing (a) write-1 current (b) write-0 current in conventional SRAM Cell and Cell with switched capacitor 
Figure 8(a) and (b) shows the variation of read current and leakage current as supply voltage is reduced from $1.1 \mathrm{~V}$ to $0.8 \mathrm{~V}$ in both the conventional SRAM cell and cell with switched capacitor, as expected the read current and leakage current falls as the supply voltage is reduced,also the plot of the read current in proposed technique is always lower than that of a conventional SRAM cell.
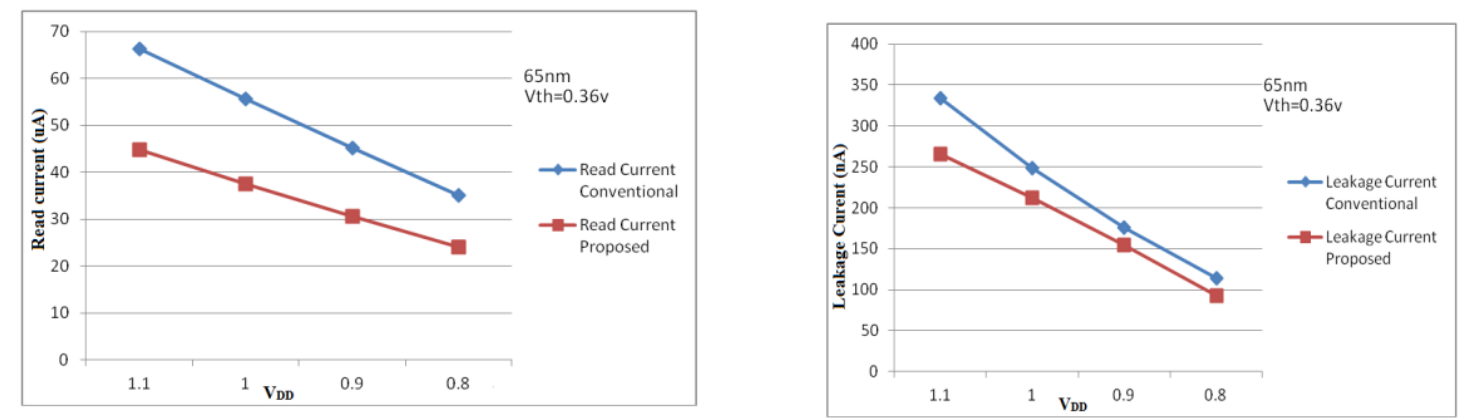

Figure 8 : Comparing (a) Read current (b) Leakage current in conventional SRAM Cell and Cell with switched capacitor

\subsection{Stability :}

Noise Margin (NM) is the maximum spurious signal that can be accepted by the device when used in a system while still maintaining the correct operation [7].the proposed technique tends to rise the source potential of the NMOS transistor of the cell and as stated in [1] source biasing tends to reduce the read noise margin thus it becomes necessary to test the cell for the stability. As shown in Figure 9(a) and (b) the static noise margin is $0.24 \mathrm{v}$ for the conventional SRAM cell and same for cell with switched capacitor circuit, thus proving that there is no effect on the SNM of the cell due to applied technique, also shown in Figure 10(a) and (b) is the read noise margin which is $0.1 \mathrm{v}$ for both the conventional SRAM cell and cell with switched capacitor.
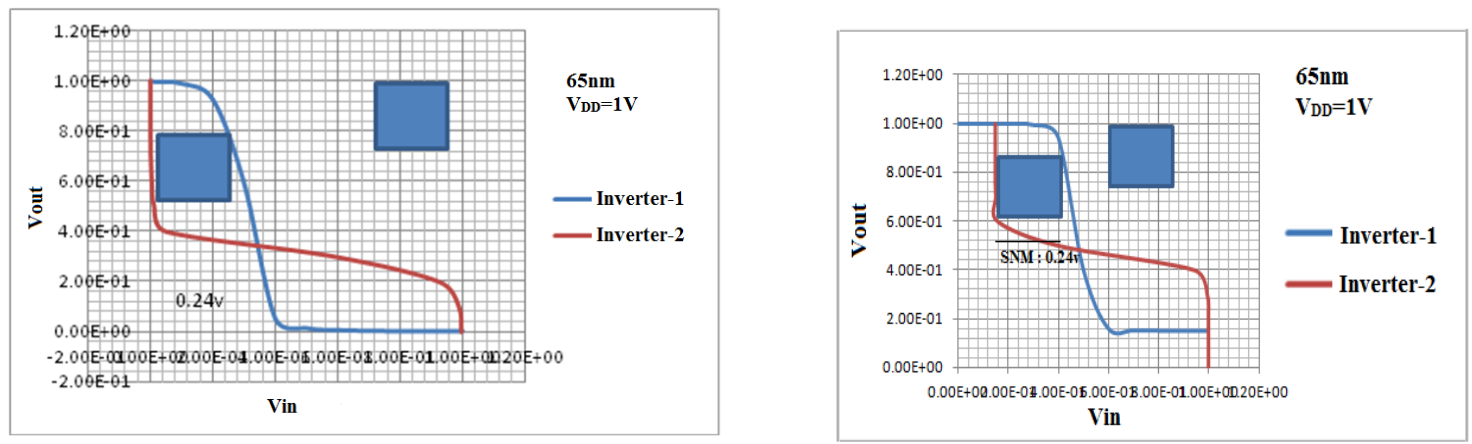

Figure 9: SNM of (a)Conventional 6T SRAM cell (b) 6T SRAM cell with switched capacitor

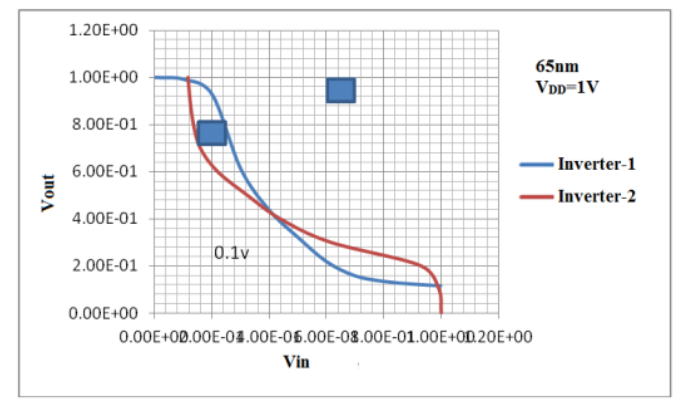

(a)

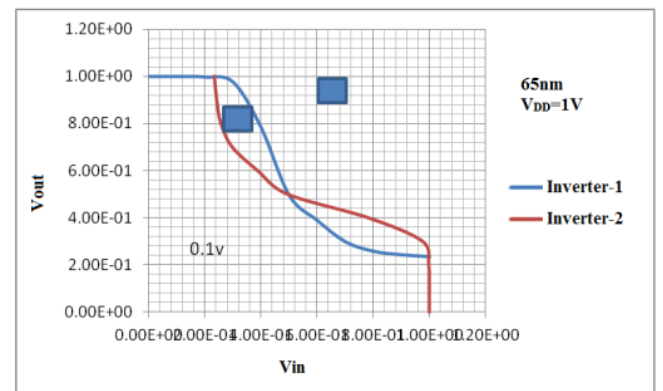

(b)

Figure 10: Read Noise margin for (a) Conventional 6T SRAM cell (b) for 6T SRAM Cell with Switched Capacitor.

Table 1 shows the read write and leakage currents at VDD $=1 \mathrm{v}$, which shows a reduction of $15.24 \%$ in write- 1 current $17.7 \%$ in write- 1 current, $32.48 \%$ in read current and $14.6 \%$ in leakage current.

Table 1: Comparing various current's in Conventional SRAM Cell and SRAM cell with proposed technique. 


\begin{tabular}{|l|l|l|l|l|}
\hline $\begin{array}{l}65 \mathrm{~nm}, \mathrm{~V}_{\mathrm{DD}}=1 \mathrm{~V}, \\
\text { Vth=0.36V }\end{array}$ & Write-1(uA) & Write-0(uA) & $\operatorname{Read}(\mathrm{uA})$ & Leakage(nA) \\
\hline Conventional SRAM & 30.83 & 30.7 & 55.57 & 249.01 \\
\hline Proposed Technique & 26.13 & 25.26 & 37.52 & 212.57 \\
\hline$\%$ Improvements & $15.24 \%$ & $17.7 \%$ & $32.48 \%$ & $14.6 \%$ \\
\hline
\end{tabular}

\subsection{NBTI Analysis}

It can be observed in Figure 11 in conventional SRAM cell the stress on transistor 4 during write-1 and read- 1 cycle is $0 \mathrm{~V}$ for $6 \mathrm{~ns}$ similarly in write- 0 and read-0 cycles the stress is $1 \mathrm{~V}$ for $9 \mathrm{~ns}$ and in idle phase the stress is $1 \mathrm{~V}$ for $3 \mathrm{~ns}$,it means out of total $15 \mathrm{~ns}$ of operation transistor- 4 will face a stress voltage of $0 \mathrm{~V}$ for $40 \%$ of time and a stress voltage of $1 \mathrm{~V}$ for remaining $60 \%$,now if we observe same conditions in proposed technique we can see that for first $6 \mathrm{~ns}$ the stress on transistor is $0 \mathrm{~V}$ but in the next $3 \mathrm{~ns}$ as the switched capacitor gets charged the potential at $\mathrm{Q}$ terminal rises to $0.2 \mathrm{~V}$ instead of $0 \mathrm{~V}$ thus reducing the stress voltage on transistor- 4 to $0.8 \mathrm{~V}$,now when the cell goes in the idle state unlike conventional cell the proposed cell is driven by the switched capacitor replacing the supply voltage of $1 \mathrm{~V}$ by the switched capacitor voltage of $0.65 \mathrm{~V}$, thus again reducing the stress voltage to $0.65 \mathrm{~V}$ instead of $1 \mathrm{~V}$ thus now of the total $15 \mathrm{~ns}$ of time the time for which the stress voltage is $1 \mathrm{~V}$ is reduced to $20 \%(3 \mathrm{~ns} / 15 \mathrm{~ns})$ of total time and stress voltage is $0.8 \mathrm{~V}$ for $20 \%(3 \mathrm{~ns} / 15 \mathrm{~ns}$ ) similarly $0.67 \mathrm{~V}$ for $20 \%(3 \mathrm{~ns} / 15 \mathrm{~ns})$ and $0 \mathrm{~V}$ for $40 \%$. Thus the stress is reduced from $1 \mathrm{~V}$ for $60 \%$ of total time to $1 \mathrm{~V}$ for $20 \%$ time $0.8 \mathrm{~V}$ and $0.65 \mathrm{~V}$ for $20 \%$ each, thus reducing the Vth degradation.

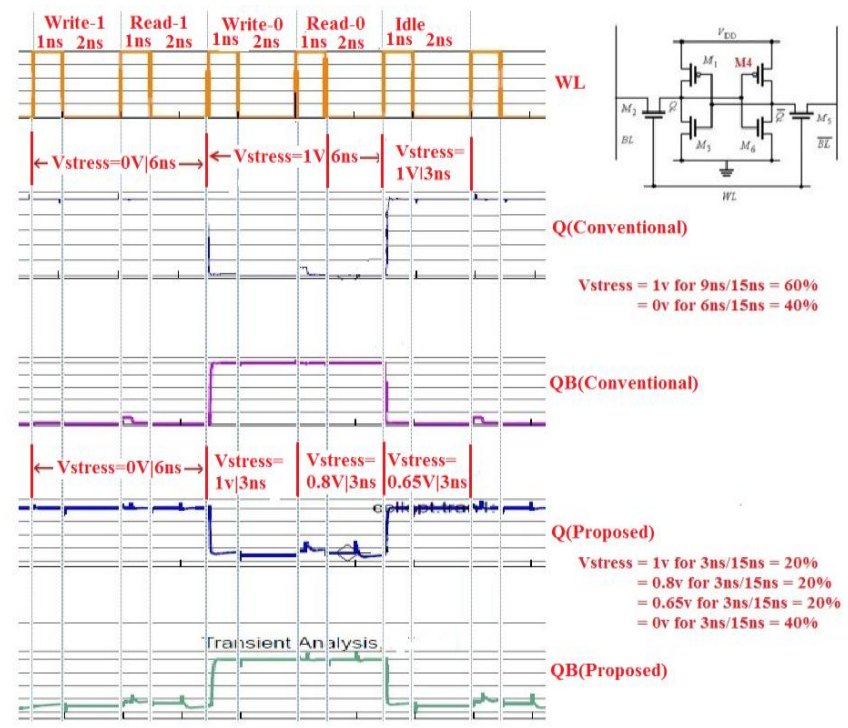

Figure 11 : Stress time for conventional and proposed SRAM cell.

Figure 12(a) shows the degradation in threshold voltage Vth with increasing temperature. As the temperature increases the bonds break more easily and the NBTI effect worsens seen in Figure 12(a).The effect of stress voltage over the Threshold voltage degradation is shown in Figure 12(v) which shows increasing rate of degradation due to increased level of stress.
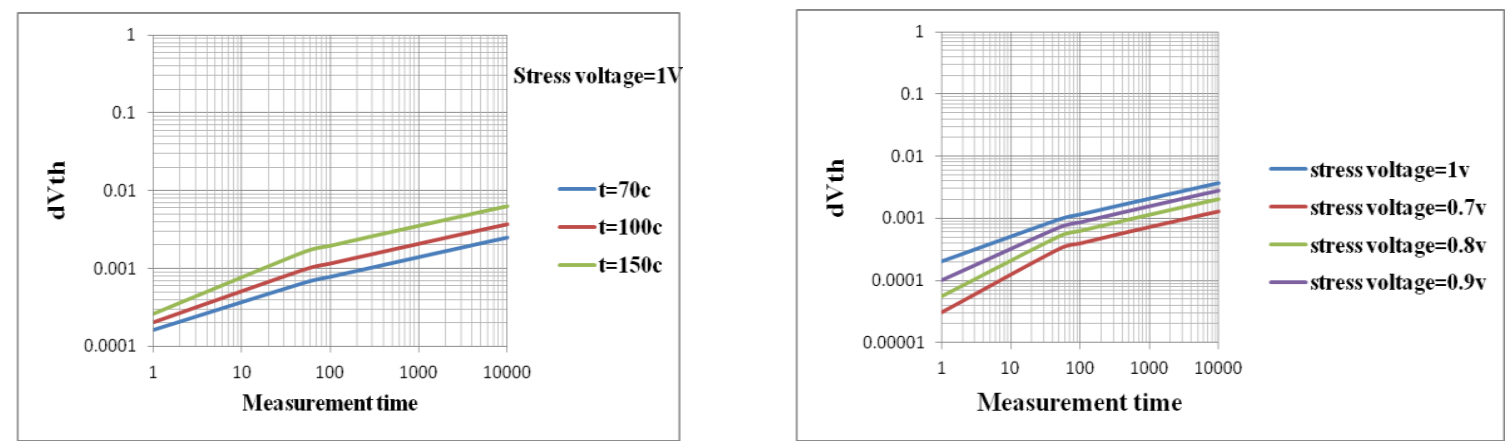

Figure 12 : dvth vs time at (a) Different temperatures (b) Different stress voltages 
Figure 13(a) shows the degradation in threshold voltage in the proposed technique, as discussed above the stress voltage over the two PMOS devices is continuously $1 \mathrm{~V}$ for $60 \%$ of total time in conventional cell whereas in proposed technique the stress reduces to $0.8 \mathrm{~V}$ for $20 \%$ of the time and again when the cell goes to idle phase the stress is reduced to $0.65 \mathrm{~V}$,these phases can be clearly observed in Figure 13(a) where the curve is continuously increasing when the stress is $1 \mathrm{~V}$ starts decreasing when the stress is $0.8 \mathrm{~V}$ and again decreases further when the stress is $0.67 \mathrm{~V}$.

It is quite clear from Figure 13(b) the difference in the rate of degradation of the two PMOS devices in conventional cell and the proposed cell, the upper curve shows the rate of degradation in conventional SRAM cell, as the stress is continuously $1 \mathrm{~V}$ the curve is also increasing continuously, whereas in the proposed technique the stress reduces from $1 \mathrm{~V}$ to $0.8 \mathrm{~V}$ to $0.67 \mathrm{~V}$ as explained above thus resulting in less rate of degradation.
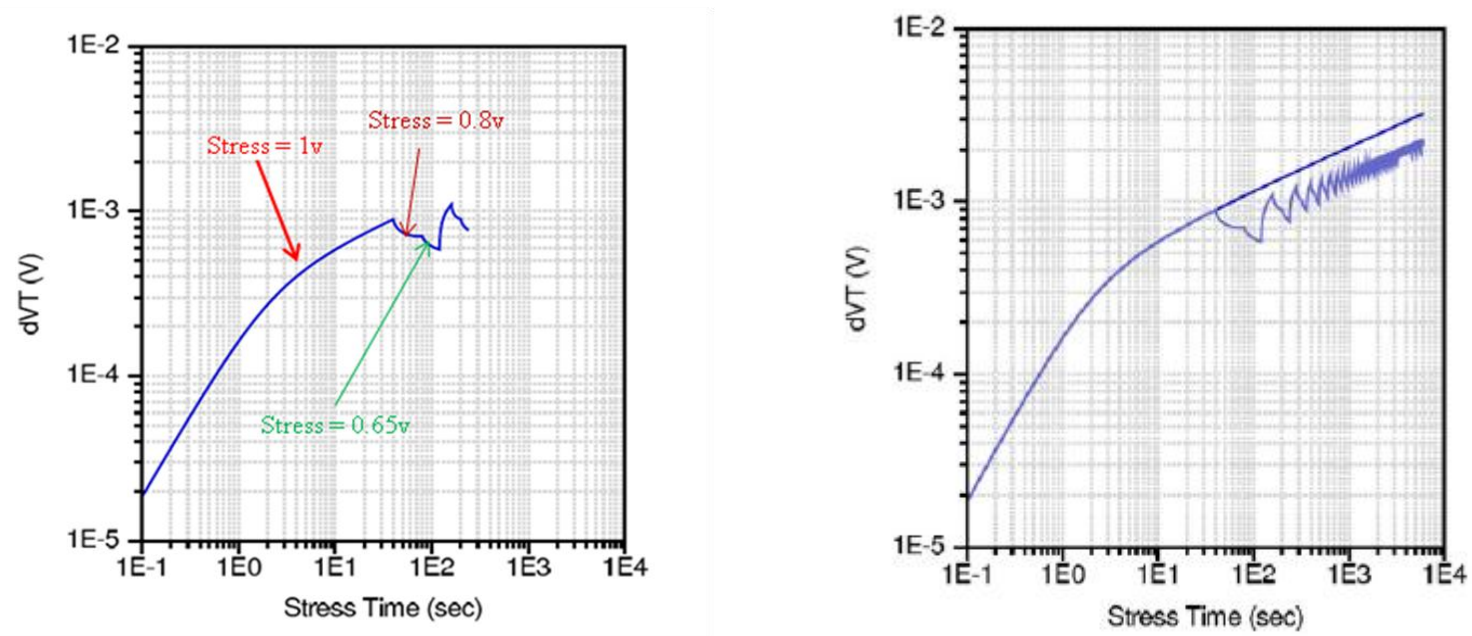

Figure 13 : (a) dvth vs time in proposed technique (b) Vth variation in conventional SRAM cell and Proposed SRAM cell

Table 2 shows the comparison of degradation in threshold voltage after 6000sec, in conventional SRAM cell where the degradation is $3.23 \mathrm{mV}$ that in proposed technique it is reduced to $2.10 \mathrm{mV}$, thus proving the effectiveness of the proposed technique in mitigating NBTI effects.

Table 2: Comparison of rate of degradation in conventional SRAM cell and proposed SRAM cell

\begin{tabular}{|l|c|c|}
\hline & $240 \mathrm{~s}$ & $6000 \mathrm{~s}$ \\
\hline $\mathrm{dV}_{\text {th }}$ in conventional SRAM cell & $1.4 \mathrm{mV}$ & $3.23 \mathrm{mV}$ \\
\hline $\mathrm{dV}_{\text {th }}$ in proposed cell & $0.76 \mathrm{mV}$ & $2.10 \mathrm{mV}$ \\
\hline
\end{tabular}

\section{Conclusion}

A novel switched capacitor based SRAM Cell is proposed in this research work. A thorough power analysis of the cell indicates a significant reduction in overall power consumption of the cell. It can be seen from the obtained results that the proposed technique reduces the Read power by $32 \%$ write power by $15 \%$ and leakage by $14 \%$ without affecting the stability of the cell even during standby mode. Also from the stability analysis it can be observed that even though the source potential is raised there is negligible variation in stability. The basic idea to provide NBTI tolerance is that if the stress is reduced the rate of Vth degradation also reduces thus as the switched capacitor is connected between the cell and the ground terminal, as the capacitors gets charged the source potential raises thus reducing the stress on both the PMOS devices, also when the cell goes to standby mode the switched capacitor circuit charged to a potential of $0.65 \mathrm{~V}$ will drive the cell, further reducing the stress. The effect can be seen in the results obtained. The degradation in threshold voltage observed in conventional cell after $6000 \mathrm{sec}$ is found to be $3.23 \mathrm{mV}$ whereas in the proposed technique the same reduces to $2.10 \mathrm{mV}$. Thus the switched capacitor technique is found to be very effective in mitigating NBTI effects while maintaining low power operation in 6T-SRAM cell. Hence, the proposed SRAM cell is suitable for low power applications in hand held devices. 


\section{References}

[1]. Swaroop Ghosh and Kaushik Roy,"Parameter Variation Tolerance and Error Resiliency:New Design Paradigm for the Nanoscale Era",proceedings of the IEEE,PP : 1718-1751,2010.

[2]. Dieter K. Schroder, "Negative bias temperature instability: What do we understand?" Microelectronics Reliability, Volume 47, Issue 6, June 2007, Pages 841-852,ISSN 0026-2714, 10.1016/j.microrel.2006.10.006.

[3]. Rakesh Vattikonda, Wenping Wang Yu Cao, "Modeling and Minimization of PMOS NBTI Effect for Robust Nanometer Design", DAC 2006, San Francisco, California, USA. July 24-28, 2006.

[4]. Taniya Siddiqua and Sudhanva Gurumurthi, "Recovery Boosting: A Technique to Enhance NBTI Recovery in SRAM Arrays", IEEE Annual Symposium on VLSI,2010.

[5]. Himani Kaushal, Eswaran.p, "Multi-SRAM Reduction Power Through Recovery Boosting", International Conference on Devices, Circuits and Systems (ICDCS),43,15-16 March 2012.

[6]. N.K shukla, R.K.Singh, Manisha Pattanaik."Design and Analysis of a Novel Low-Power SRAM Bit-Cell Structure at Deep-SubMicron CMOS Technology for Mobile Multimedia Applications".(IJACSA) International Journal of Advanced Computer Science and Applications, Vol. 2, No.5,2011.

[7]. Andrei Pavlov, Manoj Sachdev,"CMOS SRAM Circuit Design and Parametric Test in Nano-Scaled Technologies", Auburn USA,ch:1-3,PP,1-77,2011.

[8]. Saibal Mukhopadhyay, Hamid Mahmoodi, Kaushik Roy."Modeling of Failure Probability and Statistical Design of SRAM Array for Yield Enhancement in Nanoscaled CMOS.IEEE transactionson computer aided design of integrated circuits and systems, vol24,no.12,dec-2005.

[9]. Hanh-Phuc Le, Seth R. Sanders, Elad Alon."Design Techniques for Fully Integrated Switched-Capacitor DC-DC Converters",IEEE Journal of Solid State Circuits, VOL.46, NO.9, September 2011.

[10]. Masood Qazi, Sinangil, Mahmut E, Chandrakasan, Ariantha P."Challenges and Directions for Low-Voltage SRAM".IEEE Design \& Test of Computers,January/February 2011.

[11]. Keejong Kim, Mahmoodi, H. Roy, K."A Low-Power SRAM Using Bit-Line Charge-Recycling", IEEE Journal of Solid-State Circuits, VOL. 43, NO. 2, FEBRUARY 2008.

[12]. Jaideep p kulkarni, Kim Keejong, Park,Sang Phill, Roy, Kairshik."Process Variation Tolerant SRAM Array for Ultra Low voltage Applications", DAC 2008, Anaheim,California USA, pp 108-113,june 8-13,2008.

[13]. Krisztian Flautner, Nam Sung Kim, Steve Martin, David Blaauw, Trevor Mudge," Drowsy Caches: Simple Techniques for Reducing Leakage Power", ISCA 2002.

[14]. Byung-Do Yang,"A Low-Power SRAM Using Bit-Line Charge-Recycling for Read and Write Operations", IEEE Journal of SolidState Circuits, VOL. 45, NO. 10, OCTOBER 2010.

[15]. Singhal, V.K.; Singh, B., "64-bits low power CMOS SRAM by using 9T cell and charge recycling scheme," Devices, Circuits and Systems (ICDCS), 2012 International Conference on , vol., no., pp.29,33, 15-16 March 2012.

[16]. Kunhyuk Kang,Haldun Kuuoglu,Kaushik Roy and Muhammad Ashraful Alam,"Impact of Negative-Bias Temperature Instability in Nanoscale SRAM Array: Modeling and Analysis",IEEE transactions on computer aided design of integrated circuits and systems, VOL. 26, NO. 10,october 2007.

[17]. Paridhi Athe,S. Dasgupta,"A Comparative Study of 6T, 8T and 9T Decanano SRAM cell",2009 IEEE Symposium on Industrial Electronics and Applications (ISIEA 2009), October 4-6, 2009.

[18]. M.A. Alam, H. Kuuoglu, D. Varghese, S. Mahapatra, A comprehensive model for PMOS NBTI degradation: Recent progress, Microelectronics Reliability, Volume 47, Issue 6, June 2007, Pages 853-862, ISSN 0026-2714, 10.1016/j.microrel.2006.10.012.

[19]. M.A. Alam, S. Mahapatra, A comprehensive model of PMOS NBTI degradation, Microelectronics Reliability, Volume 45, Issue 1, January 2005, Pages 71-81,ISSN 0026-2714, 10.1016/j.microrel.2004.03.019.

[20]. Rakesh Vattikonda ,Wenping Wang Yu Cao, "Modeling and Minimization of PMOS NBTI E_ect for Robust Nanometer Design", DAC 2006, San Francisco, California, USA. July 24-28, 2006.

[21]. A. E. Islam, H. Kufluoglu, D. Varghese, S. Mahapatra, and M. A. Alam. "Recent Issues in Negative Bias Temperature Instability: Initial Degradation, Field- Dependence of Interface Trap Generation, and Hole Trapping Effects and Relaxation" Special Issue on Modeling of Nanoscale Transistors (Invited),IEEE Trans-action of Electron Devices, 54(9), pp. 2143-2154, 2007. 